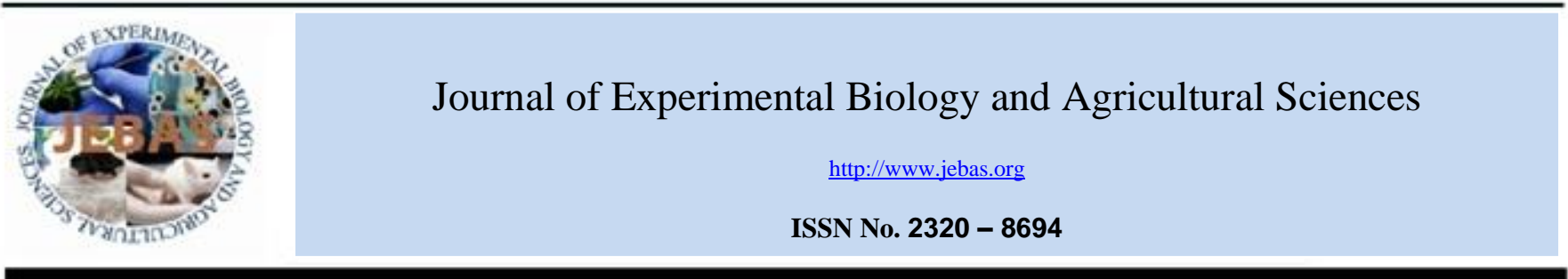

\title{
INDIGENOUS WILD EDIBLE FRUITS: SUSTAINABLE RESOURCES FOR FOOD, MEDICINE AND INCOME GENERATION - A STUDY FROM NAGALAND, INDIA
}

\section{Neilazonuo Khruomo; Chitta Ranjan Deb*}

Department of Botany, Nagaland University, Lumami 798627, Nagaland, India

Received - December 31, 2017; Revision - March 20, 2018; Accepted - April 23, 2018

Available Online - April 25, 2018

DOI: http://dx.doi.org/10.18006/2018.6(2).405.413

KEYWORDS
Alternative food
Forest products
Income generation
Market acceptability
Underutilized fruits
Sustainability

\begin{abstract}
Indigenous Wild Edible Fruits (IWEF) plays a vital role in the daily life of the rural people as they provide sustainable resources. The present study aimed at the documentation of IWEF's of three districts of Nagaland, India viz., Kohima, Phek and Tuensang and assessment of market acceptability. A total of 47 IWEF's belonging to 29 family and 39 genera were collected and identified. Market survey was carried out to check the market acceptability of the collected IWEF's in these three districts of Nagaland. Rhus. semialata, T. chebula, S. pinnata, D. indica, E. officinalis, F. semicordata, E. floribundus, J. regia, M. esculenta, P. pershia are some fruits that are common used by the local inhabitants and some of these fruits are also used to treat different diseases through traditional methods.
\end{abstract}

* Corresponding author

E-mail: debchitta@ rediffmail.com; debchitta@gmail.com (Chitta Ranjan Deb)

Peer review under responsibility of Journal of Experimental Biology and Agricultural Sciences.

Production and Hosting by Horizon Publisher India [HPI] (http://www.horizonpublisherindia.in/).

All rights reserved.
All the article published by Journal of Experimental Biology and Agricultural Sciences is licensed under a Creative Commons Attribution-NonCommercial 4.0 International License Based on a work at www.jebas.org.

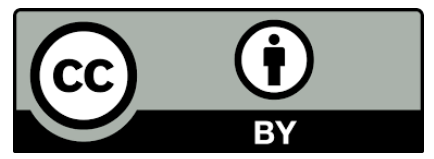




\section{Introduction}

Plants for human consumption account for $\sim 5 \%$ of the total plant species of the world (Asfaw \& Tadesse, 2001). Forest has a large and indispensable role in improving the food security and livelihood of the tribal society (Yesodharan \& Sujana, 2007). During early days, man lived by hunting and fruit gathering collected from the wild (Tomar et al., 2015). Since, wild edible plants are freely accessible within natural habitats; indigenous people have more knowledge in gathering and preparing food items from these wild plant resources (Somnasang \& MorenoBlack, 2000). Fruits being a major forest product, supplement human diet as they provide essential vitamins, minerals and fiber required for maintaining health (Kumari, 2008). They play a significant role in the wide range of agricultural system as a source of wild food and have an important socio-economic role through their uses in medicine, dyes, shelter, fibers, and religious and traditional ceremonies (FAO, 1999). Fruits being one of largest forest resource have the potentiality to uplift the economic condition as well as providing the food security to the local people of the region (Deb et al., 2013). World over, tribal population stores a vast knowledge on utilization of local plants as food and other specific uses (Sudriyal et al., 1998). A large number of wild spices used by the tribal in meeting their daily requirement are through the diverse vegetation of that area. Use of large number of wild species by the tribal to meet their diverse requirement is largely due to the prevalence of diversity of vegetation in the area (Katewa, 2003). The North-Eastern region of India is inhabited by mixed tribes and mostly dominated by the tribal people unlike the mainland of India. Nagaland state is situated in the North-Eastern part of India and is a hilly area surrounded by dense natural forest with warm and cool climatic condition and variation in rainfall thereby making it one of the richest and diverse flora and fauna in the North-East region of India. Nagaland state is inhabited mostly by the tribal's with distinct dialects and cultural features. Utilization of wild edible plants as a food source is an integral part of the culture of indigenous people of Naga tribe as the forest harbors rich and unique biodiversity with the state being a part of the Indo-Burma biodiversity hot spot (Deb et al., 2016). Since early times, edible wild fruits have played a very vital part in supplementing the diet of the people and to meet their basic need of food, mostly the tribal people, and some of which are preserved for use in dry period or sold in rural market (Deshmukh \& Waghmode, 2011). They also form an additional income (for small landholders and landless) living near forest and fringes through sale in local market (Pradheep et al., 2016). The market price fluctuates according to the season of collection, climate and availability. A vendor earns their livelihood from the selling of these forest resources, thereby sustaining their livelihood - INR 1 lakh to 2.5 lakhs for a season (Sashimatsung et al., 2013). Some of the commonly abundant fruits and fruit products found in the household as well as the local market are Rhus semialata, Terminalia chebula, Spondias pinnata, Docynia indica, Emblica officinalis, Ficus semicordata, Elaeocarpus floribundus, Juglans regia, Myrica esculenta, Pyrus pershia, Castanopsis indica Choerospondias axillaris, Diospyros kaki, Hodgsonia macrocarpa etc. Wild fruits collection is not a gender oriented in the region, both young and old, men and women are equally involved or participate. Indigenous Wild Edible Fruits (IWEF) contributes immensely to the nutrition of the local inhabitants of Nagaland. Present study was undertaken to document the IWEF of three districts of Nagaland, India viz., Kohima, Phek and Tuensang, assess the market acceptability as a source of 'sustainable resources for food, medicine and income generation'.

\section{Materials and Methods}

\subsection{Survey areas}

The district of Kohima, Phek and Tuensang of Nagaland was considered for the present study. Kohima is the capital city of Nagaland located between $25.40^{\circ} \mathrm{N}$ and $94.08^{\circ} \mathrm{E}$ and is surrounded by the Assam state to the west, Zunheboto district to the east, Wokha district to the north and Manipur state to the south. It is the home of the Angamis Naga tribe and agriculture is the main occupation. Phek district lies in the South-East of Nagaland located between $25.40^{\circ} \mathrm{N}$ and $94.28^{\circ} \mathrm{E}$ bounded by Kohima district in the west, Zunheboto and Kiphire districts in the North, Myanmar in the South East and Manipur state in the South. The district is inhibited by the Chakhesang Naga tribe and agriculture is the main occupation with $80.84 \%$ of the population engaged in agriculture. While, Tuensang district is the largest district of Nagaland located between $26.14^{\circ} \mathrm{N}$ and $94.49^{\circ} \mathrm{E}$. The district shares an international border with Myanmar all along its eastern sector and is bounded by Mon district in the north east, Longleng in the north, Mokokchung in the south. Chang, Sangtam, Yimchunger and Khiamniungan are the main indigenous tribes of the district. Jhum is the main agricultural practice of the district. Figure 1 showing the three study areas viz., Kohima, Phek and Tuensang district.

\subsection{Field work/data collection}

Intensive field works were undertaken along with the local field guide in different fruiting seasons in the districts of Kohima, Phek and Teunsang during the year 2014-2016. The primary aim of the study is to collect, identify and document the wild edible fruits used by the local inhabitants of these districts of Nagaland. The authentic identification of the collected wild fruits was done with the help of the available authentic literature and also the experts in the concern fields. Information based on the mode of consumption and uses as food or medicines by the locals were also recorded by interacting with the local inhabitants. Both conventional and 


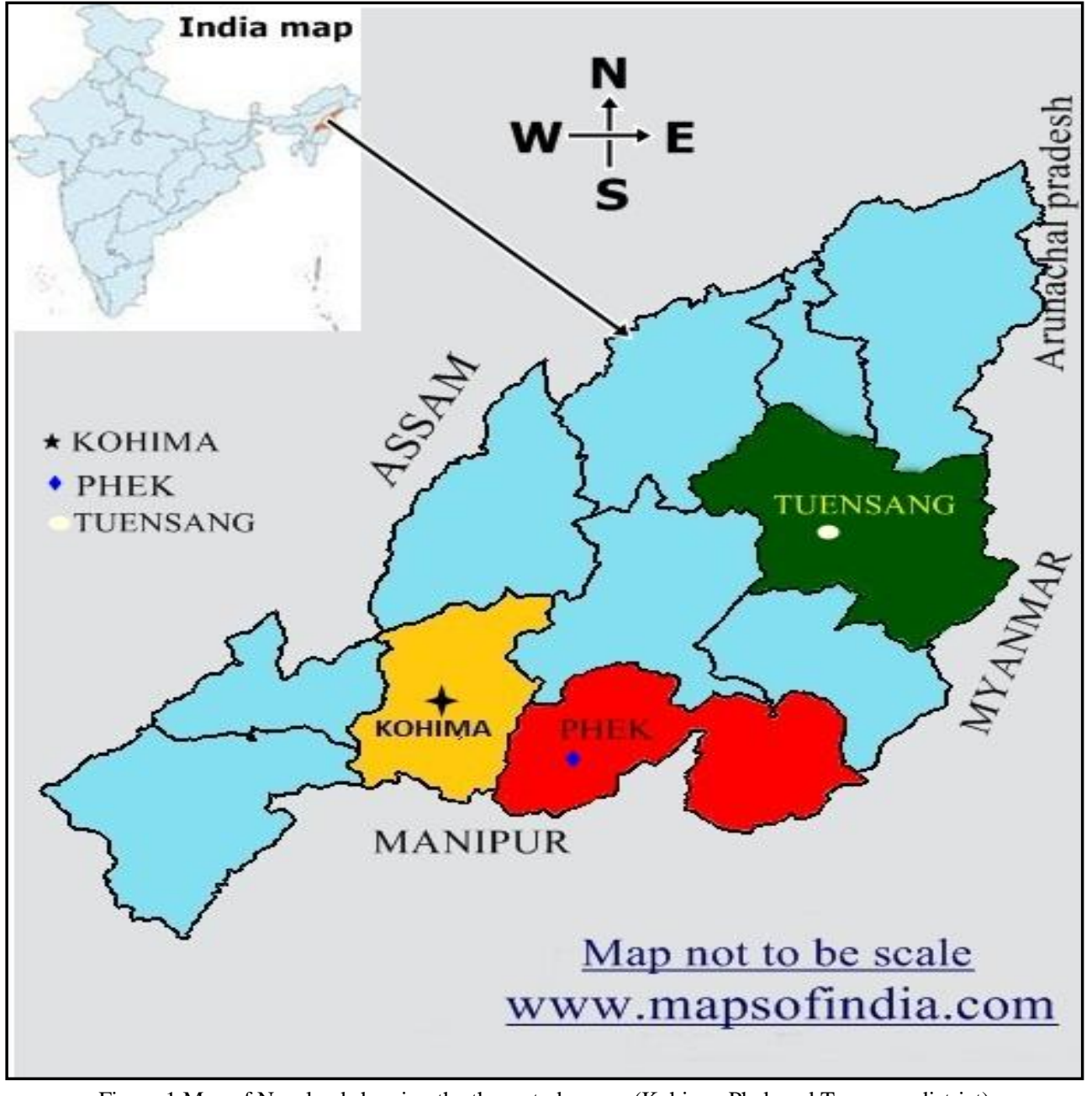

Figure 1 Map of Nagaland showing the three study areas (Kohima, Phek and Tuensang district)

digital herbariums were maintained for future study. The collected fruits were preserved in $2 \%$ formalin $(\mathrm{v} / \mathrm{v})$ in the jar bottles and deposited in the Department of Botany, Nagaland University for future references. Market survey was also carried out in the local market of these districts to check the market acceptability of the indigenous wild edible fruits. During the market survey, the marketed plants and their product were recorded along with their marketed rate/price as the products are sold either in packets, plate, bunch or cup (local system of marketing). There is no specify standard measurement units exist as the price varies according to different seasons of the collection, area to area etc.

\section{Results}

In the present study, a total of 47 IWEFs have been collected belonging to 29 family and 39 genera (Table 1). The collected IWEF belongs to the family Actinidiaceae, Anacardiaceae,
Berberidaceae, Caesalpiniaceae, Cannabaceae, Capparaceae, Combretaceae, Cornaceae, Clusiaceae, Cucurbitaceae, Ebenaceae, Elaeocarpaceae, Euphorbiaceae, Fagaceae, Juglandiaceae, Lauraceae, Moraceae, Myricaceae, Myrtaceae, Olacaceae, Oxalidaceae, Passifloraceae Phyllantheceae, Rhamnaceae, Rhizophoraceae, Rosaceae, Rutaceae, Solanaceae, Urticaceae. The family Rosaceae exhibits the maximum number with 8 species (18\%) followed by the family Actinidiaceae Anacardiaceae, Cucurbitaceae, Moraceae with 3 species each (7.2\%). The distribution of families is shown in table 2. Some of common fruits are shown in figure 2. The collected plants are arranged in alphabetical order with their common name, family, flowering and fruiting season and mode of consumption along with their accession number (Table 1). Table 3 shows some of IWEF's used in different traditional methods of food and medicine by the inhabitants of these three districts. Some of the fruit products were found to be sold fresh, dried or cooked in the 
Table 1: List of Indigenous Wild Edible Fruits of three districts of Nagaland

\begin{tabular}{|c|c|c|c|c|}
\hline Scientific name \& family & Common name & $\begin{array}{l}\text { Flowering \& } \\
\text { Fruiting }\end{array}$ & $\begin{array}{l}\text { Mode of } \\
\text { consumption }\end{array}$ & Accession No. \\
\hline $\begin{array}{l}\text { Aphananthe cuspidata } \\
\text { (Blume) Planch. (Cannabaceae) }\end{array}$ & Aphananthe & March-September & $\begin{array}{l}\text { Ripped fruit } \\
\text { eaten raw }\end{array}$ & NU-BOT-UCK-NK-10056 \\
\hline $\begin{array}{l}\text { Actinidia callosa Lindl. } \\
\text { (Actinidiaceae) }\end{array}$ & Wild kiwi & May-July & $\begin{array}{l}\text { Ripped fruit } \\
\text { eaten raw }\end{array}$ & NU-BOT-UCK-NK-10050 \\
\hline $\begin{array}{l}\text { Antidesma bunius } \\
\text { (L.) Spreng. (Phyllanthaceae) }\end{array}$ & Antidesma & April-August & $\begin{array}{l}\text { Ripped fruit } \\
\text { eaten raw }\end{array}$ & NU-BOT-UCK-NK-1002 \\
\hline $\begin{array}{l}\text { Bauhinia variegate } \\
\text { Linn. (Caesalpiniaceae) }\end{array}$ & Bauhinia & February-May & $\begin{array}{l}\text { Immature fruit } \\
\text { eaten fresh }\end{array}$ & NU-BOT-UCK-NK-10023 \\
\hline $\begin{array}{l}\text { Carallia brachiata (Lour.) } \\
\text { Merr. (Rhizophoraceae) }\end{array}$ & Carallia & October-April & $\begin{array}{l}\text { Mature fruit } \\
\text { taken raw }\end{array}$ & NU-BOT-UCK-NK-10065 \\
\hline $\begin{array}{l}\text { Castanopsis indica } \\
\text { (Roxb. ex Lindl.) A.DC. (Fagaceae) }\end{array}$ & Chestnut & April-December & $\begin{array}{l}\text { Mature nuts is } \\
\text { roasted or } \\
\text { eaten raw }\end{array}$ & NU-BOT-UC-NK-10041 \\
\hline $\begin{array}{l}\text { Choerospondias axillaris } \\
\text { (Roxb.) B.L Burtt \& A.W. } \\
\text { (Anacardiaceae) }\end{array}$ & Hill Hog plum & January-June & $\begin{array}{l}\text { Ripped fruit } \\
\text { eaten raw }\end{array}$ & NU-BOT-UC-NK-10018 \\
\hline $\begin{array}{l}\text { Cucumis hystrix } \\
\text { Chakrav. (Cucurbitaceae) }\end{array}$ & Wild Cucumber & $\begin{array}{l}\text { September- } \\
\text { December }\end{array}$ & $\begin{array}{l}\text { Young fruits } \\
\text { eaten raw }\end{array}$ & NU-BOT-UC-NK-10030 \\
\hline $\begin{array}{l}\text { Debregeasia longifolia } \\
\text { (Burm.f.) Wedd.(Urticaceae) }\end{array}$ & Orange Wild Rhea & June-November & $\begin{array}{l}\text { Ripped fruit } \\
\text { eaten raw }\end{array}$ & NU-BOT-UCK-NK-10062 \\
\hline $\begin{array}{l}\text { Cornus capitata Wall } \\
\text { (Cornaceae) }\end{array}$ & Evergreen dogwood & May-December & $\begin{array}{l}\text { Mature fruit } \\
\text { eaten raw }\end{array}$ & NU-BOT-UC-NK-10086 \\
\hline $\begin{array}{l}\text { Diospyros kaki L.F } \\
\text { (Ebenaceae) }\end{array}$ & Asian persimmon & August-December & $\begin{array}{l}\text { Mature fruit } \\
\text { eaten raw }\end{array}$ & NU-BOT-UC-NK-10027 \\
\hline $\begin{array}{l}\text { Docynia indica } \\
\text { (Wall.) Decne. (Rosaceae) }\end{array}$ & Wild apple & February-October & $\begin{array}{l}\text { Mature fruit } \\
\text { eaten raw }\end{array}$ & NU-BOT-UC-NK-10032 \\
\hline $\begin{array}{l}\text { Elaeocarpus floribundus } \\
\text { Blume (Elaeocarpaceae) }\end{array}$ & Indian olive & March-September & $\begin{array}{l}\text { Mature fruit } \\
\text { eaten raw }\end{array}$ & NU-BOT-UC-NK-10083 \\
\hline $\begin{array}{l}\text { Eleaegnus latifolia } \mathrm{L} . \\
\text { (Elaeagnaceae) }\end{array}$ & Wild olive & November-June & $\begin{array}{l}\text { Ripped fruit } \\
\text { eaten raw }\end{array}$ & NU-BOT-UC-NK-10024 \\
\hline $\begin{array}{l}\text { Emblica officinalis Gaertn. } \\
\text { (Euphorbiaceae) }\end{array}$ & Goose berry & March-April & $\begin{array}{l}\text { Mature fruit } \\
\text { eaten raw or } \\
\text { dried }\end{array}$ & NU-BOT-UC-NK-10070 \\
\hline $\begin{array}{l}\text { Tetradium fraxinifolium } \\
\text { (Hook.f.) T.G. Hartley (Rutaceae) }\end{array}$ & - & May-November & $\begin{array}{l}\text { Mature fruit } \\
\text { taken as spice }\end{array}$ & NU-BOT-UC-NK-10057 \\
\hline $\begin{array}{l}\text { Ficus hispida Linn. } \\
\text { (Moraceae) }\end{array}$ & Wild fig & June-October & $\begin{array}{l}\text { Ripped fruit } \\
\text { eaten raw }\end{array}$ & NU-BOT-UC-NK-10069 \\
\hline $\begin{array}{l}\text { Ficus racemosa Linn. } \\
\text { (Moraceae) }\end{array}$ & Fig & January-July & $\begin{array}{l}\text { Mature fruit } \\
\text { eaten raw }\end{array}$ & NU-BOT-UC-NK-10011 \\
\hline $\begin{array}{l}\text { Ficus semicordata } \\
\text { Buch-Ham ex J.E. Smith } \\
\text { (Moraceae) }\end{array}$ & Drooping fig & May-October & $\begin{array}{l}\text { Mature fruit } \\
\text { eaten raw }\end{array}$ & NU-BOT-UC-NK-10012 \\
\hline $\begin{array}{l}\text { Garcinia xanthochymus } \\
\text { Hook.f. ex T.Anderson (Clusiaceae) }\end{array}$ & Sour mangosteen & March-July & $\begin{array}{l}\text { Ripped fruit } \\
\text { eaten raw }\end{array}$ & NU-BOT-UC-NK-10044 \\
\hline
\end{tabular}




\begin{tabular}{|c|c|c|c|c|}
\hline Scientific name $\&$ family & Common name & $\begin{array}{l}\text { Flowering \& } \\
\text { Fruiting }\end{array}$ & $\begin{array}{c}\text { Mode of } \\
\text { consumption }\end{array}$ & Accession No. \\
\hline $\begin{array}{l}\text { Hodgsonia macrocarpa } \\
\text { (Blume) Cogn. (Cucurbitaceae) }\end{array}$ & Lard Seed & March-May & $\begin{array}{l}\text { Ripped fruit } \\
\text { eaten after }\end{array}$ & NU-BOT-UC-NK-10063 \\
\hline $\begin{array}{l}\text { Hovenia dulcis Thunberg } \\
\text { (Rhamnaceae) }\end{array}$ & Japanese raisin & May-June & $\begin{array}{l}\text { Mature pedicles } \\
\text { eaten raw }\end{array}$ & NU-BOT-UC-NK-10087 \\
\hline $\begin{array}{l}\text { Juglans regia Linn. } \\
\text { (Juglandaceae) }\end{array}$ & Local walnut & March-October & $\begin{array}{l}\text { Mature fruit } \\
\text { eaten raw }\end{array}$ & NU-BOT-UC-NK-10013 \\
\hline $\begin{array}{l}\text { Litsea cubeba (Lour.) Pers. } \\
\text { (Lauraceae) }\end{array}$ & Mountain pepper & $\begin{array}{l}\text { December- } \\
\text { February }\end{array}$ & $\begin{array}{l}\text { Mature fruit } \\
\text { taken as spice }\end{array}$ & NU-BOT-UC-NK-10064 \\
\hline $\begin{array}{l}\text { Mahonia nepaulensis DC. } \\
\text { (Berberidaceae) }\end{array}$ & Blue berry & October-April & $\begin{array}{l}\text { Ripped fruit } \\
\text { eaten raw }\end{array}$ & NU-BOT-UC-NK-10035 \\
\hline $\begin{array}{l}\text { Myrica esculenta } \\
\text { Buch-Ham ex. D. Don (Myricaceae) }\end{array}$ & Box-myrtle & January-May & $\begin{array}{l}\text { Ripped fruit } \\
\text { eaten raw }\end{array}$ & NU-BOT-UC-NK-10036 \\
\hline $\begin{array}{l}\text { Olax imbricata Roxb. } \\
\text { (Olacaceae) }\end{array}$ & Olax & April-October & $\begin{array}{l}\text { Ripped fruit } \\
\text { eaten raw }\end{array}$ & NU-BOT-UC-NK-10071 \\
\hline $\begin{array}{l}\text { Oxalis corniculata Linn. } \\
\text { (Oxalidaceae) }\end{array}$ & Wood Sorrel & February-August & $\begin{array}{l}\text { Young fruit } \\
\text { eaten raw }\end{array}$ & NU-BOT-UC-NK-1008 \\
\hline $\begin{array}{l}\text { Passiflora sulpeltata } \\
\text { Ortega (Passifloraceae) }\end{array}$ & Wild granadilla & October-May & $\begin{array}{l}\text { Ripped fruit } \\
\text { eaten raw }\end{array}$ & NU-BOT-UC-NK-10090 \\
\hline $\begin{array}{l}\text { Physalis peruviana } \mathrm{L} . \\
\text { (Solanaceae) }\end{array}$ & Cape goose berry & October-January & $\begin{array}{c}\text { Ripped fruit } \\
\text { eaten raw }\end{array}$ & NU-BOT-UC-NK-10037 \\
\hline $\begin{array}{l}\text { Prunus carmesina H. Hara } \\
\text { (Rosaceae) }\end{array}$ & Wild cherry & March-June & $\begin{array}{l}\text { Ripped fruit } \\
\text { eaten raw }\end{array}$ & NU- BOT-UC-NK-10053 \\
\hline $\begin{array}{l}\text { Prunus nepaulensis (Ser.) } \\
\text { Steud. (Rosaceae) }\end{array}$ & - & April-August & $\begin{array}{l}\text { Ripped fruit } \\
\text { eaten raw }\end{array}$ & NU-BOT-UC-NK-10043 \\
\hline $\begin{array}{l}\text { Pyrus pashia } \text { Buch-Ham. } \\
\text { Ex D. Don (Rosaceae) }\end{array}$ & Himalayan pear & March-October & $\begin{array}{l}\text { Mature fruit } \\
\text { eaten raw }\end{array}$ & NU-BOT-UC-NK-10085 \\
\hline $\begin{array}{l}\text { Rhus semialata Murr. } \\
\text { (Anacardiaceae) }\end{array}$ & Nutgall Tree & August-January & $\begin{array}{l}\text { Mature fruit } \\
\text { eaten raw }\end{array}$ & NU-BOT-UC-NK-10014 \\
\hline $\begin{array}{l}\text { Rubus ellipticus } \mathrm{Sm} . \\
\text { Rosaceae (Rosaceae) }\end{array}$ & Golden Raspberry & February-April & $\begin{array}{l}\text { Ripped fruit } \\
\text { eaten raw }\end{array}$ & NU-BOT-UC-NK-10040 \\
\hline $\begin{array}{l}\text { Rubus indotibetanus Koidz. } \\
\text { (Rosaceae) }\end{array}$ & - & March-May & $\begin{array}{l}\text { Ripped fruit } \\
\text { eaten raw }\end{array}$ & NU-BOT-UC-NK-10068 \\
\hline $\begin{array}{l}\text { Rubus niveus Thunb. } \\
\text { (Rosaceae) }\end{array}$ & Hill raspberry & April-June & $\begin{array}{l}\text { Ripped fruit } \\
\text { eaten raw }\end{array}$ & NU-BOT-UC-NK-10039 \\
\hline $\begin{array}{l}\text { Saurauia macrotricha } \\
\text { Kurt ex Dyer (Actinidiaceae) }\end{array}$ & Saurauia & May-August & $\begin{array}{l}\text { Ripped fruit } \\
\text { eaten raw }\end{array}$ & NU-BOT-UC-NK-10059 \\
\hline $\begin{array}{l}\text { Saurauia punduana Wall. } \\
\text { (Actinidiaceae) }\end{array}$ & Saurauia & April-August & $\begin{array}{l}\text { Ripped fruit } \\
\text { eaten raw }\end{array}$ & NU-BOT-UC-NK-10058 \\
\hline $\begin{array}{l}\text { Spondias pinnata } \\
\text { (L. f.) Kurz (Anacardiaceae) }\end{array}$ & Wild mango & March-May & $\begin{array}{l}\text { Ripped fruit } \\
\text { eaten raw }\end{array}$ & NU-BOT-UC-NK-10020 \\
\hline Stixis suaveolens & Stixis & September-April & $\begin{array}{c}\text { Mature fruits } \\
\text { eaten raw }\end{array}$ & NU-BOT-UC-NK-10019 \\
\hline $\begin{array}{l}\text { Syzygium cumini (L.) } \\
\text { Skeels (Myrtaceae) }\end{array}$ & Black Plum & February-August & $\begin{array}{l}\text { Ripped fruit } \\
\text { eaten raw }\end{array}$ & NU-BOT-UC-NK-10042 \\
\hline $\begin{array}{l}\text { Terminalia bellerica } \\
\text { (Gaertn.) Roxb. (Combretaceae) }\end{array}$ & Bastard myrobalan & March-July & $\begin{array}{l}\text { Mature nuts } \\
\text { eaten raw }\end{array}$ & NU-BOT-UC-NK-10067 \\
\hline $\begin{array}{l}\text { Terminalia chebula Retz. } \\
\text { (Combretaceae) }\end{array}$ & Myrobalan & April-July & $\begin{array}{l}\text { Mature fruit } \\
\text { eaten raw }\end{array}$ & NU-BOT-UC-NK-10015 \\
\hline $\begin{array}{l}\text { Toddalia asiatica (L.) Lam } \\
\text { (Rutaceae) }\end{array}$ & Orange climber & February-May & $\begin{array}{l}\text { Ripped fruit } \\
\text { eaten raw }\end{array}$ & NU-BOT-UC-NK-10060 \\
\hline $\begin{array}{l}\text { Trichosanthes dunniana } \\
\text { H. Lév. (Cucurbitaceae) }\end{array}$ & Trichosanthes & July-November & $\begin{array}{l}\text { Ripped fruit } \\
\text { eaten raw }\end{array}$ & NU-BOT-UC-NK-10066 \\
\hline
\end{tabular}



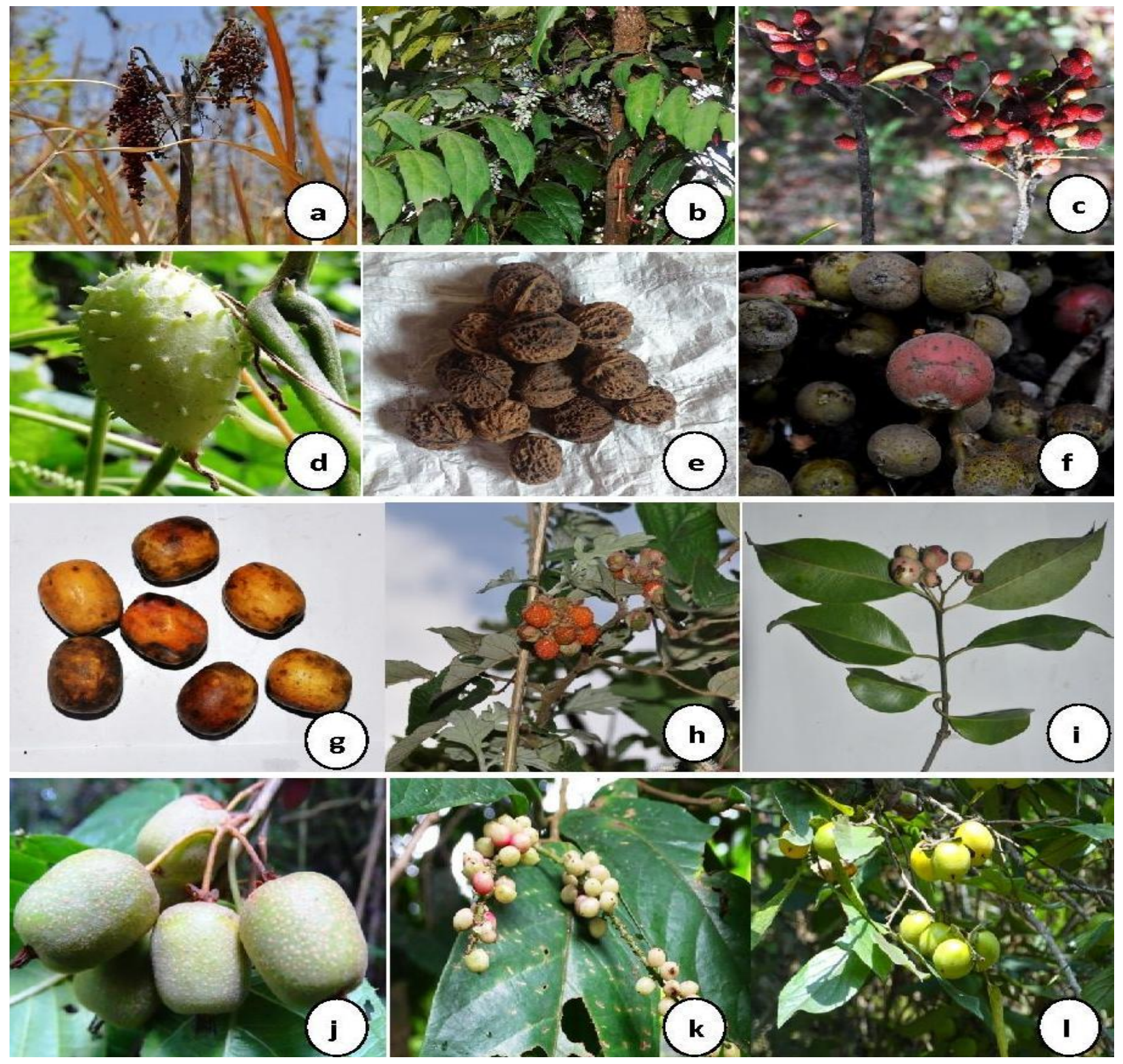

Figure 2 Some of the collected IWEF. a. Rhus semialata Murr., b. Mahonia nepaulensis DC., c. Myrica esculenta Buch-Ham ex. D. Don, d. Cucumis hystrix Chakrav., e. Juglans regia Linn., f. Ficus semicordata Buch-Ham ex J.E. Smith, g. Choerospondias axillaris (Roxb.) B.L Burtt \& A.W. Hill, h. Rubus ellipticus Sm., i. Syzygium cumini (L.) Skeels, j. Actinidia callosa Lindl., k. Antidesma bunius (L.) Spreng., 1. Diospyros kaki L.F.

local market during the market survey. Table 4 shows some of the indigenous wild fruits in rate per unit INR that were found selling in the local market of the three study area. The market price or rate of the fruits varies from area to area, from season to season and also supply of the product in the market by the locals.

The market price of the product ranges from INR 10-100 per packet/cup/plate. The market price also varies in all the local markets of these districts. During the market survey in the local market of these three districts, it was found that there was no standard measurement exists for the products that are marketed. The market survey shows that some of these fruits sold are highly accepted and demanded due to its good taste and medicinal value.

\section{Discussion}

In India especially the North-Eastern region, the majority of the rural communities are dependent on the forest product to meet their food needs even during food crisis. The diverse biodiversity of Nagaland plays an important and significant role in providing

Journal of Experimental Biology and Agriculture Science http://www.jebas.org 
Table 2 Family distribution pattern of collected fruits during field survey

\begin{tabular}{|c|c|c|c|c|c|}
\hline Family & No. of species & $\begin{array}{c}\text { Distribution } \\
\text { Frequency }(\%)\end{array}$ & Family & No. of species & $\begin{array}{c}\text { Distribution } \\
\text { Frequency (\%) }\end{array}$ \\
\hline Actinidiaceae & 3 & 7.1 & Lauraceae & 1 & 2.1 \\
\hline Anacardiaceae & 3 & 7.1 & Moraceae & 3 & 7.1 \\
\hline Berberidaceae & 1 & 2.1 & Myricaceae & 1 & 2.1 \\
\hline Caesalpiniaceae & 1 & 2.1 & Myrtaceae & 1 & 2.1 \\
\hline Cannabacea & 1 & 2.1 & Olacaceae & 1 & 2.1 \\
\hline Capparaceae & 1 & 2.1 & Oxalidaceae & 1 & 2.1 \\
\hline Combretaceae & 2 & 4.1 & Passiflorace & 1 & 2.1 \\
\hline Cornaceae & 1 & 2.1 & Phyllantheceae & 1 & 2.1 \\
\hline Clusiaceae & 1 & 2.1 & Rhamnaceae & 1 & 2.1 \\
\hline Cucurbitaceae & 3 & 7.1 & Rhizophoraceae & 1 & 2.1 \\
\hline Ebenaceae & 1 & 2.1 & Rosaceae & 8 & 18 \\
\hline Elaeocarpaceae & 1 & 2.1 & Rutaceae & 2 & 4.1 \\
\hline Euphorbiaceae & 1 & 2.1 & Solanaceae & 1 & 2.1 \\
\hline Fagaceae & 1 & 2.1 & Urticaceae & 1 & 2.1 \\
\hline Juglandiaceae & 1 & 2.1 & & & \\
\hline
\end{tabular}

Table 3 Some IWEP's used in traditional methods of food and medicine

Botanical Name

Choerospondias axillaris

(Roxb.) B.L Burtt \& A.W.

Hill

Juglans regia Linn.

Rhus semialata Murr.

Terminalia chebula Retz.

Spondias pinnata (L. f.)

Kurz

Ficus semicordata Buch-

Ham ex J.E. Smith

Emblica officinalis Gaertn.

Docynia indica (Wall.)

Decne.

Hodgsonia macrocarpa

(Blume) Cogn.

Litsea cubeba (Lour.) Pers.

Elaeocarpus floribundus

Blume

Stixis suaveolens

(Roxburgh) Pierre

Bauhinia variegata $\mathrm{Linn}$.

Castanopsis indica (Roxb.

ex Lindl.) A.DC.

\section{Traditional Food and Medicine}

Mature fruit is dried and cooked as vegetable. Bark water extract is use to treat second degree burn.

Nut is roasted to make pickle from the kernel. Oil from fruit is used in Cough, weakness in legs, dysentery and constipation

Fruit is grinded into powder which is used in some local made sweets. Powder taken during indigestion, stomachache, and food poisoning by Ao-Naga tribes

Fruit is slightly cooked with salt, chili and dried and eaten. Dried or raw fruits used in stomach disorders, pneumonia, gastric and indigestion.

Dried fruit is taken as vegetable, pickle is made from the mature fruit. Fruit juice is taken by diabetic patients.

Mature fruit is use to make juice, decoction of bark is used for diabetes.

Mature fruit is use to make juice, fruits dried and eaten. Fruit juice is used in dysentery, conjunctivitis, constipation, piles and is a natural source of vitamin $\mathrm{C}$.

Mature fruit is use to make juice, fruits dried and eaten. Fruit used against cold and cough. Leaves used in dysentery and diarrhea.

Seed is roasted or baked and eaten, also used to make pickle. Decoction of leave is drank to treat fever and also for ulcer.

Fruit is used as spice and used in making pickle. Powdered root and bark is used to relieve pain.

Mature fruit slightly cooked with salt/sugar and dried and eaten

The seed is remove from the seed coat and slightly cooked with salt, chili and dried it and eaten.

Young raw pods used in making salad or mix with pickle and eaten.

Nuts is roasted with salt and eaten as snack along with red tea. 
Table 4 Some indigenous wild fruits sold in the local market of Kohima, Phek and Tuensang district.

\begin{tabular}{|c|c|}
\hline Name of Plant & Rate per unit* in INR \\
\hline Bauhinia variegata Linn & $20-50 /$ plate or packet \\
\hline Castanopsis indica (Roxb. ex Lindl.) A.DC. & 20-50/packet \\
\hline Choerospondias axillaris (Roxb.) B.L Burtt \& A.W. Hill & $10-20 /$ plate or cup \\
\hline Docynia indica (Wall.) Decne. & 20-50/plate or packet \\
\hline Elaeocarpus floribundus Blume & $20-50 /$ plate or cup or packet \\
\hline Elaeagnus latifolia $\mathrm{L}$. & 20-50/plate \\
\hline Emblica officinalis Gaertn & 20-50/plate or packet \\
\hline Ficus semicordata Buch-Ham ex J.E. Smith & 20-30/plate \\
\hline Hodgsonia macrocarpa (Blume) Cogn. & 20-50/plate or packet \\
\hline Juglans regia Linn. & 50-100/cup or packet \\
\hline Litsea cubeba (Lour.) Pers & 20-50/plate or packet \\
\hline Prunus nepalensis (Ser.) Steud. & $20-50 /$ bunch or plate \\
\hline Rhus semialata Murr. & $20-50 /$ cup or packet \\
\hline Spondias pinnata (L. f.) Kurz & 20-50/plate or cup \\
\hline Stixis suaveolens (Roxburgh) Pierre & 20-50/plate or packet \\
\hline Terminalia chebula Retz. & 20-50/packet \\
\hline
\end{tabular}

Note: No standard measurement system exists. Units for sale approximately only: $* 1$ plate or cup or packet $=$ Approx. $500 \mathrm{gm}$.

various wild edible plants specially people living in the core remote areas that faces lesser opportunities and chances as compare to those living in the town. Since the forest resources are freely accessible to the local, wild edible fruits become an important source of food and nutrient to the locals. The uses of wild edibles fruits are different among the tribes of Nagas. They have their use of tradition food and medicine which are passed down from generation to generation. The present study shows that both young and old indulge in collecting IWEF's for various purposes such as food, medicine and marketing. Some of the collected fruits are documented in figure 2. The market survey shows that the plants and products are well accepted in the local markets of these districts. The market rate also varies from area to area, season to season collection and abundance and availability of the plants and plant products in the local market (Deb et al., 2016).

Collection and utilization of IWEF's has become an important part in the daily lives of the rural people as they represent a cheaper and easy accessible and also offering opportunities for income generation to the local people uplifting the socioeconomic status of the local people. But with the passage of time, the rich biodiversity of the state is facing extensive threats due to ever growing population leading to more demand for land and space, forest fires, unplanned urbanization, forest products and timber collection, 'Jhum cultivation' and many anthropogenic activities. Therefore, proper strategy needs to be implemented for conservation of the forest to prevent extinction of plants in the near future. Many plants which are considered underutilized or neglected are important crops at national and regional level; therefore availability of information on plant species with potentiality for food, medicine and income generation should be promoted through sustainable collection and trade which will enable resource development and better management of these plants. Wild edible fruits contribute significantly to the nutritional security of mankind across the world (Bhatt et al., 2017). Many collected fruits have been used for traditional medicine purposed by the locals (Table 3), therefore phytochemical and nutraceutical studies of these wild edible fruits will provide better nutritional source for the future. Also, many local products have been produce from the wild fruits that has been collected but their product outputs may be missing for various reasons, therefore it is the need of hour for the policy and decision makers to take initiative steps to improve the processing of the local products, to create awareness for better market acceptability, proper commercial scale cultivation, proper conservation strategies (Deb et al., 2016) and promote them to global level. Wild edible plant persists as they are in use by the local people in different means. 


\section{Conclusion}

The present study is an approach made to promote the wild edible plants that are richly available in the rural areas to the higher level (regional, national and also global) and an attempt to widen the shrinking food basket which mankind has been relying upon for generations. At the same time, taking in consideration that they be use judiciously so it sustains the near future generation as the study shows that these plants are growing under pressure from various anthropogenic activities. Therefore, public awareness and community based management needs to be encouraged as well. Research on indigenous fruits should also be taken up and disseminate the results so to have diversity in diet.

\section{Acknowledgement}

Authors are thankful to the Department of Biotechnology, Ministry of Science and Technology, New Delhi, India for financial support for the present study through 'Institutional Biotech Hub to Prof. C. R. Deb. Authors are also thankful to the local people of Kohima, Phek and Tuensang for providing their valuable knowledge and rendering their services during the survey. Facilities used from the UGC-SAP(DRS-III) programme is duly acknowledged.

\section{Declaration}

Authors declare that there is no conflict of interest exists. Further, declare that the manuscript has not been published in any journal/book or proceedings or in any other publication or offered for publication elsewhere in substantially the same or abbreviated form, either in print or electronically.

\section{References}

Asfaw Z, Tadesse M (2001) Prospect for sustainable use and Development of Wild Food Plants in Ethiopia. Economic Botany 55: 47-62.

Bhatt ID, Rawat S, Bhandhani A, Rawal RS (2017) Nutraceutical potential of selected wild edible fruits of the Indian Himalayan region. Food Chemistry 215: 84-91.

Deshmukh BS, Waghmode A (2011) Role of wild edible fruits as a food resource: Traditional knowledge. International Journal of Pharmacy \& Life Sciences 2: 919-924.

Deb CR, Jamir NS, Ozukum S (2013) A study on the survey and documentation of Underutilized crops of three district of Nagaland, India. Global Journal of Bioscience 2: 67-70.

Deb CR, Khruomo N, Jamir NS (2016) A study on documentation and market acceptability of Underutilized Wild Edible Crops of Kohima district, Nagaland, India. Asian Journal of Biology and Life Sciences 5: 201-206.

FAO Publications (1999) Use and potential of wild plants, Information division and food and agricultural organization of the united nation, Rome, Italy.

Kumari A (2008) Nutritional quality, functional properties and value addition of underutilized fruits of Himachal Pradesh, $\mathrm{PhD}$ Thesis submitted to the Himachal Pradesh Krishi Vishvavidyalaya, Himachal Pradesh, India).

Katewa SS (2003) Contribution of some wild food plant from the forestry to the diet of tribals of southern Rajasthan. Indian Forester 129: 1117-1131.

Pradheep K, Soyimchiten, Pandey A, Bhatt KC (2016) Wild edible plants used by Konyak tribe in mon district of Nagaland: Survey and inventorisation. Indian Journal of Natural Product and Resources 7:74-81.

Somnasang P, Moreno-Black G (2000) Knowing, gathering and eating: knowledge and attribute about wild food in an Asian village in North-eastern Thailand. Journal of Ethnobiology 20: 197.

Sashimatsung M, Giribabu, Lanusunep (2013) A study on marketable surplus and price spread of tomato in Mokokchung district, Nagaland. International Journal of Humanities and Social Science invention 2:37-42.

Sundriyal M, Sundriyal RC, Sharma E, Porohit AN (1998) Wild edible and other useful plants from the Sikkim Himalaya, India. Oecologia Montana 7:43-54.

Tomar A, Kumar A, Dubey K (2015) Underutilized wild edible fruits of nutritional and medicinal value. Journal of Research Education in Indian Medicine 21: 1-8.

Yesodharan K, Sujana KA (2007) Wild edible plants traditionally used by the tribes of Parambikulam Wildlife Sanctuary, Kerala, India. Natural Product Radiance 6: 74-80. 